\title{
Separation and purification of bio-diesel : A review
}

\author{
Chaocheng Zheng ${ }^{1,2, a}$ \\ ${ }^{1}$ Nanjing Communications Institute of Technology, Nanjing 211188, China; \\ ${ }^{2}$ Jiangsu province transportation energy saving engineering technology research center, Nanjing \\ 211188, China.
}

Author E-mail: zccnau@126.com

Keywords: biofuel; separation; purification; membrane technology

\begin{abstract}
Bio-diesel-a biodegradable, sustainable and clean energy, has received growing interest especially in recent years, chiefly on account of development in biodiesel fuel and serious ecological pressures nowadays. In the production process of biodiesel from biomass, separation and purification is a critical technology. Conventional technologies utilized for separation such as gravitational settling, decantation, filtration and biodiesel purification have been proven to be inefficient, energy consumptive, and less cost effective as well. The involvement of membrane reactor and separative membrane shows huge promise for separation and purification of biodiesel. Hence, membrane technology needs to be explored and exploited to overcome these difficulties usually encountered in the separation and purification of biodiesel. In this paper both conventional and most recently membrane technologies used in refining biodiesel have been critically reviewed. The effects of catalysts, free fatty acids, water content and oil to methanol ratios on the purity and quality of biodiesel are also examined.
\end{abstract}

\section{Introduction}

Increasing demand for clean energy, global warming and fast diminishing supply of fossil fuels are major key factors leading to people's search for alternative sources of energy. Till now, some of the most notable alternative sources of energy were mostly water, solar, wind energy, and biofuels. Currently, 86 percent of the energy being consumed worldwide and nearly $100 \%$ of energy desired in the transportation sector is provided by non-renewable fossil fuels[1]. Biofuels production is being supported by European Union (EU) with the ultimate aim of increasing fuel supply sources, boosting decarbonisation of fuels for transportation, decreasing hazardous gaseous emission which causes global warming effects, thus, providing more earning opportunities in rural communities and developing long term plans for finite fossil fuels replacement. Currently, several countries such as USA, Germany, Brazil, Australia, Italy, and Austria are already using biofuels such as biodiesel and bioethanol[2]. Since the passage of the Energy Policy Act of 2005, biodiesel use has been increasing in the United States. In the UK, the Renewable Transport Fuel Obligation obliges suppliers to include $5 \%$ renewable fuel in all transport fuel sold in the UK by 2010. For road diesel, this effectively means $5 \%$ biodiesel.

Biodiesel (usually composing of fatty acid alkyl esters, FAAE), a substitute to diesel fuel, is often produced from renewable natural sources such as vegetable oils, animal fats and microalgal oils. It is biodegradable, sustainable, and moreovber, also environmentally beneficial, thereby providing lower gas emission profile. Biodiesel is considered to be carbon neutral, as biodiesel yielding plants such as jatropha curcas, rape plant and palm trees to absorb carbon-dioxide to a greater extent than that being contributed to the atmosphere when used as fuel in diesel engines. Also, biodiesel has similar physicochemical properties to that of diesel produced from crude oil and can be used directly to run existing diesel engines without major modifications or as a mixture with petroleum diesel and produces less harmful gas emission such as sulfur oxide. However, the direct use of vegetable oils as fuel in compression ignition engines is problematic due to their high viscosity and low volatility. These oil types do not burn completely and form carbon deposits in the fuel injectors of diesel engines. The viscosity of vegetable oils can be better improved with transesterification reaction, a process 
which seems to insure very good outcomes in terms of lowering viscosity and enhancing other physicochemical properties[3]. Transesterification is a chemical reaction involving triglycerides and an alcohol of lower molecular weights using homogeneous or heterogeneous substances as catalyst to yield biodiesel and glycerol. In the history, transesterification of a vegetable oil was conducted as early as 1853 by Patrick Duffy, four decades before the first diesel engine became functional. Rudolf Diesel's prime model, a single $10 \mathrm{ft}(3.0 \mathrm{~m})$ iron cylinder with a flywheel at its base, ran on its own power for the first time in Augsburg, Germany, on 10 August 1893 running on nothing but peanut oil. In remembrance of this event, 10 August has been declared "International Biodiesel Day.

Even though transesterification reaction catalyzed by alkali homogeneous catalyst such as sodium and potassium hydroxides yields higher conversion of vegetable oil to methyl esters in short time, the reaction has several shortshormings: it is energy intensive; recovery of glycerol is difficult; the catalyst has to be removed from the product; alkaline wastewater requires treatment and free fatty acids (FFA) and water interfere with the reaction[4]. The presence of water lowers the activity of catalyst, while FFA react with the catalyst to produce saponified product[2]. The formation of soap reduces the biodiesel yield, and causes significant difficulty in product separation and purification. Thus, biodiesel and byproduct, glycerol have to be refined by washing with hot deionized water two to three times, leading to high waste of time, energy and water[3,4]. The major limiting factor to biomass use is the technology development for the separation, purification, and transformation of it into biochemicals and biofuels. Currently, "down-stream processing” alone accounts for $80 \%$ of the process costs[5]. Ineffective biodiesel separation and purification causes severe diesel engines problems such as plugging of filters, coking on injectors, more carbon deposits, excessive engine wear, oil ring sticking, engine knocking, and thickening and gelling of lubricating oil[6]. Several different separation and purification of biodiesel techniques have been studied. This paper reviews the technologies employed with emphasis on the most suitable practice for effective separation. Membrane separation seems to be the most suitable for this purpose and is the focus of this study.

\section{Conventional biodiesel separation techniques}

Several researchers had studied extensively numerous conventional techniques for the separation of biodiesel. Most of the researchers reported that high-quality biodiesel that is economically viable can be achieved when suitable biodiesel separation process is employed. After transesterification, separation of biodiesel and by-product, glycerol is usually first carried out. This process of biodiesel separation is based on the facts that the biodiesel and glycerol produced are typically sparingly mutually soluble, and that there is palpable difference in density between biodiesel and glycerol phases respectively. Moreover, this difference in density is sufficiently enough for the application of simple techniques such as gravitational settling or centrifugation for the separation of biodiesel and glycerol phases. In addition, the separation rate of biodiesel mixture is influenced by numerous factors such as intense mixing, formation of emulsion, solubility of biodiesel in glycerol, and glycerol in biodiesel[6]. Demirbas reported that supercritical methanol process is non-catalytic, involves a much simpler purification of transesterified products, has a lower reaction time, is more environmentally friendly, and requires lower energy use[7]. Another advantage with supercritical methanol is that the conversion gets $95 \%$ complete in $10 \mathrm{~min}$. Siti et al. revealed the problems encountered in the use of chemical catalysts to be high energy and methanol consumptions, and large amount of alkaline wastewater. The use of enzymes such as lipase has recently received a wider attention and considered to be an effective way to overcome such problems. Particularly, the separation of glycerol without complicated treatment. Even though cost is the major bottle neck associated with enzymatic catalysis.

Sharma et al. stated that methanol has polar hydroxyl group which can act as an emulsifier causing emulsification and rendering severe difficulties in the separation of the methyl ester layer from water. 


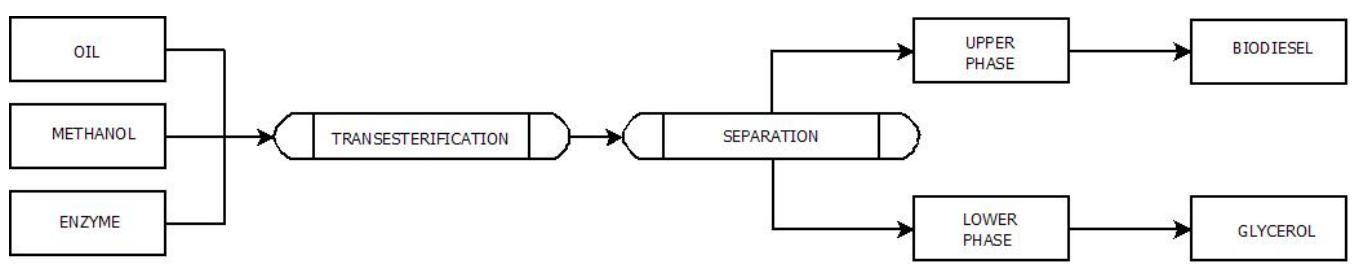

Fig.1 Enzymatic biodiesel production

\section{Conventional biodiesel purification techniques}

The main objective of biodiesel washing is to remove free glycerol, soap, excess alcohol, and residual catalyst. The drying of alkyl ester is needed to achieve the stringent limits of biodiesel specification on the amount of water content in the purified biodiesel product. However, there are other treatments used to reduce biodiesel colour, remove glycerides, sulfur and phosphorus, from the fuel. Water has the ability to provide a means for addition of acid to neutralize the unreacted alkaline homogeneous catalyst. This process simplifies immediate removal of the salt products. The unreacted methanol after transesterification reaction should be removed before the washing stage to minimize the presence of alcohol in the wastewater effluent. However, some processes remove the excess methanol after washing with water Von Gerpen et al. The authors prevented precipitation of saturated fatty acid esters using deionized water. Formation of emulsions is retarded when gentle water washing is applied fostering rapid and complete phase separation. Calcium and magnesium contamination is eliminated with the help of softened water (slightly acidic) which has the capability of neutralizing the remaining unreacted alkali catalysts. Similarly, iron and copper ions removal get rid of a source of catalysts that reduces the fuel stability[7]. Sharma et al. reported that simple biodiesel purification process and recovery of high-quality glycerin are the key factors to be considered to reduce the price of biodiesel fuel and make it competitive to the conventional diesel fuel.

Transesterification reaction is a reaction that is widely considered and mostly adopted to produce commercial biodiesel. The transesterified products undergo different purification techniques in order to purify biodiesel from glycerol and other by-products, while water washing is generally carried out to remove soap, catalyst, methanol and other contaminants from biodiesel, using deionized water.

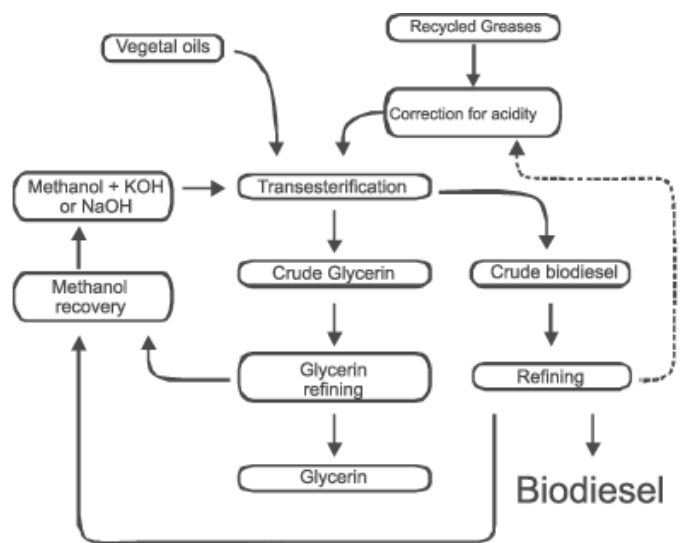

Fig.2 Schematic process for bio-diesel production

\section{Biodiesel membrane separation and purification}

The membrane equipments mostly produced for the separation and purification of crude biodiesel seem to exhibit several advantages over the conventional ones such as eradication/minimization of higher capital cost and other related costs of production, and provide high specific area of mass transfer. Membrane equipments for biodiesel refining are usually made from inorganic microporous ceramic membranes and generally have a lot of applications in biotechnology. These membranes hold 
some hope in their use for biofuels. Some of the most effective devices used for the separation and purification of crude biodiesel include: Membrane reactor and separative ceramic membrane.

\section{Conclusion}

Biodiesel is world's first Advanced Biofuel. It is a renewable, clean-burning diesel replacement that is reducing country's dependence on imported diesel, creating green jobs and improving our environment. It is made from an increasingly diverse mix of resources including agricultural oils, recycled cooking oil and animal fats and meets the strict specifications.

\section{Acknowledgments}

This work was supported by a grant from Scientific Research Project of Nanjing Communications Institute of Technology (JY1305), High-level Scientific Research Foundation for the introduction of talent in Nanjing Communications Institute of Technology, Qing Lan Project of Nanjing Communications Institute of Technology, college students' innovation and entrepreneurship training program in Jiangsu province (201412804003Y, 201512804001Y), and Higher Vocational Education Research Fund of Nanjing Communications Institute of Technology (14JY103).

\section{References}

[1] Thiam LC, Subhash B. Catalytic processes towards the production of biofuels in a palm oil and oil palm biomass-based bio-refinery[J]. Bioresource Technology 2008,99:7911-7922.

[2] Balat M, Balat H. Progress in biodiesel processing[J]. Applied Energy 2010,87:1811-1835.

[3] Ferella F, Mazziotti G, De Michelis I et al. Optimization of the transesterification reaction in biodiesel production[J]. Fuel,2010,89:36-42.

[4] Hameed B H, Lai L F, Chin L H. Production of biodiesel from palm oil (Elaeis guineensis) using heterogeneous catalyst: an optimized process[J]. Fuel Processing Technology,2009,90:606-610.

[5] Tai-Shung NC. Development and purification of biodiesel[J].Separation and Purification Technology, 2007,20:377-381.

[6] Demirbas A. Progress and recent trends in biodiesel fuels[J].Energy Conversion and Management 2009,50:14-34.

[7] Sharma Y C, Singh B, Upadhyay S N. Advancements in development and characterization of biodiesel: a review[J].Fuel,2008,8:235-273. 